\title{
NEW TECHNIQUES IN X-RAY ASTRONOMY
}

\author{
C. DE JAGER \\ (University Observatory and Space Research Laboratory, Utrecht, The Netherlands)
}

\section{ABSTRACT}

A review is given of some modern developments in X-ray astronomy. Improved designs of proportional counters and other detectors are described. We further enumerate developments in solar X-ray spectrographs and heliographs, like the 'gutter' spectrograph, and the paraboloidal-hyperboloidal frustrum mirror system, the zone plate technique. We also give a review of instruments and collimating techniques for the detection of non-solar celestial X-ray sources.

\section{Introduction; Optical and X-Ray Astronomy}

Astrophysical observational techniques are essentially based on the three following properties of astronomical information:

(a) The faintness of the photonic flux, which makes necessary the construction of large light-collecting areas with a low background noise. Only the Sun with its large flux is an exception.

(b) The smallness of the details, making necessary an angular resolution of the order of $10^{-5}$ radians.

(c) The need for detailed spectral information, necessitating a spectral resolution ranging between $\Delta \lambda / \lambda \approx 10^{-1}$ (broad-band observations, for object recognition and for statistical information), or to $\approx 10^{-3}$ (detection of strong spectral lines; orienting astrophysical investigations), or down to $\approx 10^{-5}$ (line profiles, detailed and refined astrophysical interpretation).

These properties apply to radiation of any wavelength, also to X-rays. An essential property of X-ray astronomical instruments is that refracting media can not be applied in this wavelength range, so that all imaging or spectral resolution should be based on reflection and/or diffraction. Furthermore, roughly speaking, normal reflection is impossible for wavelengths shorter than about $500 \AA$, while virtually any reflection is impossible for wavelengths below about $10 \AA$. This may easily be shown (see e.g. Mayer, 1964): If $i$ is the angle between the incident rays and the normal to the surface, we have

$$
\sin i=n=1-\delta,
$$

where the real quantity $\delta$ may be approximated as

$$
\delta \approx 2 \cdot 7 \times 10^{10} Z \rho \lambda^{2} / A .
$$

Perek (ed.), Highlights of Astronomy, 108-123. () I.A.U. 
By defining the Bragg angle or grazing incidence angle $\Theta=90-i$, one obtains the critical Bragg angle $\Theta_{c}$ above which no reflection will occur, according to:

$$
\sin \left(90-\Theta_{c}\right)=n-1-\delta
$$

From Equations (1) and (2) one derives, with $Z / A \approx \frac{1}{2}$ :

$$
\Theta_{c} \approx(2 \delta)^{1 / 2} \approx 1 \cdot 3 \times 10^{5} \rho^{1 / 2} \lambda
$$

For $\rho=8$ and $\lambda=10 \AA$ one finds with Equation (3): $\Theta_{c} \approx 1$ ․7. For $1 \AA: \Theta_{c} \approx 0.2$.

The wavelength below which normal incidence is impossible may roughly be put at about $500 \AA$. Thus one may distinguish between two different regions in astronomical $X$-ray techniques:

EUV optics: $\quad \lambda \gtrsim 500 \AA \quad$ normal incidence optics;

X-ray optics: $\left\{\begin{array}{cl}10 \lesssim \lambda \lesssim 500 \AA & \text { grazing incidence optics; } \\ \lambda \lesssim 10 \AA & \text { 'optics' based on mechanical collimators. }\end{array}\right.$

In this review we shall successively discuss the following problems:

(a) the construction of large $\mathrm{X}$-ray detectors with a low background noise;

(b) image forming techniques: (1) grazing incidence optics; (2) mechanical collimators;

(c) X-ray spectrographs;

(d) X-ray spectroheliographs.

This review should certainly not be considered as an exhaustive summary of X-ray instrumental techniques; we only wish to discuss new techniques and in doing this we prefer to emphasize some modern trends, rather than to give a complete summary of all developments nowadays underway.

\section{Detector Developments}

The kind of detectors used in X-ray astronomy depends on the energy range of the incident photons. Down to a wavelength of about $10 \AA$ phototubes are still useful. The windowless magnetic electron multipliers and the channeltrons go even to below $10 \AA$. At high photon energies, from about $0 \cdot 2 \mathrm{keV}(60 \AA)$ upward, proportional counters may also be used. For photonic energies between a few tens of keV's up to higher energies scintillation counters are the best kind of detectors. At very high energies (hundreds of MeV's) spark chambers and Cerenkov detectors come into the picture, but since this energy range deals with gamma- rather than X-ray astronomy it is outside the scope of the present discussion. Geiger tubes and ionisation chambers find application in a broad energy range. In this section we discuss only the magnetic electron multiplier, proportional and scintillation counters. We further refer to Mayer's (1964) and Boyd's (1965) review papers. 


\subsection{MAGNETIC ELECTRON MULTIPLIERS AND CHANNELTRONS}

The most successfully used version of this type of open multiplier is the Bendix windowless photon and particle detector. It finds many applications in the EUV and the soft X-region. With tungsten cathodes their lack of sensitivity to visible and near ultraviolet radiation offers another advantage.

When a very small light-collecting area is needed the channeltrons are very useful.

\subsection{PROPORTIONAL COUNTERS}

These are based on photo-ionization of the gas molecules in the tube. If the photonic energy is $E$, and if a photon loses $e$ electron-volts per ionization, one photon produces on the average $E / e$ ion-pairs. Hence, the relative statistical fluctuation in the photon current is $\sqrt{e / E}$. Normally $e \approx 30 \mathrm{eV}$; hence, the spectral resolution for $10 \AA$ photons $(=1 \mathrm{keV})$ is about $0 \cdot 2$. Experimentally Culhane $e t$ al. (1966) found a fractional standard deviation of $0 \cdot 167[E(\mathrm{keV})]^{-1 / 2}$, in the energy range $1-30 \mathrm{keV}$.

Hence, proportional counters are not suitable for spectral discrimination at wavelengths longer than about $10 \AA$. However, by a careful choice of gas filling and filters Den Boggende at Utrecht is able to prepare a so-called multi-wavelength, narrowband, X-ray solar spectrometer, consisting of six proportional counters, each sensitive to two different, narrow wavelength bands in the 1-60 $\AA$ region. Discrimination between the two bands of each counter and combination of the results from all counters make possible the determination of the solar flux in a dozen of small bands 2 to $3 \AA$ wide below $60 \AA$.

The use of proportional counters at wavelengths longer than $60 \AA$ poses a number of serious problems, since here the filter windows have to be extremely thin, necessitating both supporting grids and gas flow. At energies above about 50-100 keV these counters lose their efficiency, because the gas filling becomes transparent for photons of such energies.

The essential difficulties in the construction of large proportional counters for the detection of weak fluxes of $\mathrm{X}$-radiation are the wiring of the counters and the choice of the filters, which should prevent the gas from leaking away. If too much gas leaks away, so-called flow-counters should be used, in which a constant flow of gas replaces the losses. Furthermore, the counters should be mounted in an anti-coincidence circuit to reduce the effect of counting pulses due to cosmic radiation particles and other sources. A simple version of this kind for the detection of solar radiation below $3 \AA$ was constructed by Culhane et al. (1966) and is shown in Figure 1a. This 'pill-box' type of counter consists of a flat cylindrical tube with a window area of the order of 10 $\mathrm{cm}^{2}$. Two of these pill-boxes, one of which is windowless, are arranged back to back (Figure $1 b$ ) and circuited in anti-coincidence to reduce the influence of cosmic radiation pulses. 


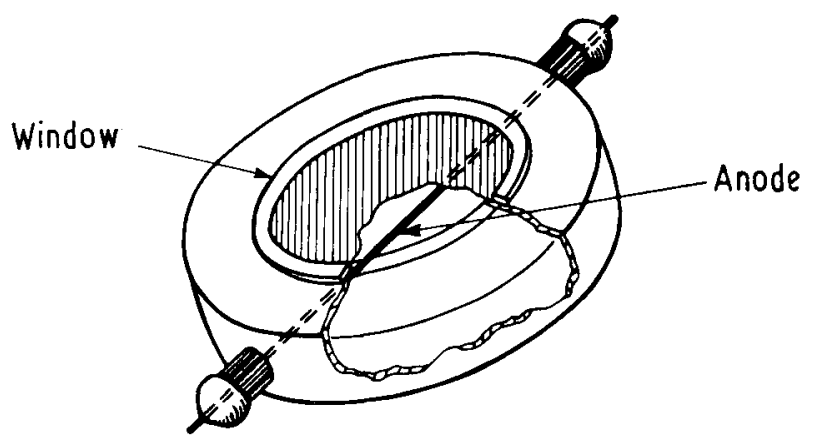

FIG. 1a. 'Pill-box' counter developed by Culhane et al. (1966), diameter $6 \mathrm{~cm}$; depth $1.2 \mathrm{~cm}$.

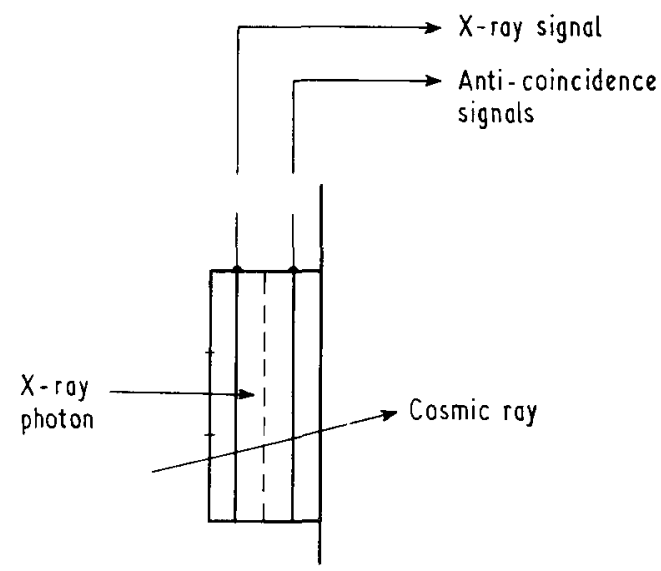

FIG. $1 \mathrm{~b}$. Operation of the double pill-box detector in anti-coincidence mode.

Proportional counters with very large areas were constructed or are under investigation by various groups. Labeyrie in Saclay produces counters with Be windows having areas of about $200 \mathrm{~cm}^{2}$. These areas can be made as large as this because the window has a considerable thickness. In other cases the areas should be made smaller, and a sufficient collecting area can then be obtained only by combining proportional counters. Thus Austin et al. (1967) at the American Science and Engineering Inc. are preparing the payload for an 'X-ray Explorer Satellite' which essentially consists of X-ray counters with dimensions of approximately $56 \times 5 \times 5 \mathrm{~cm}^{3}$. Several of these are combined to form a sensitive area of $56 \times 51 \mathrm{~cm}^{2}$. In order to reduce the background noise, these counters are combined with so-called background counters with a volume of about $56 \times 5 \times 2 \frac{1}{2} \mathrm{~cm}^{3}$; these are mounted to form a jacket around the X-ray counter, leaving only the $\mathrm{X}$-ray windows exposed to the incoming radiation from the collimators in front of the X-ray detectors. 


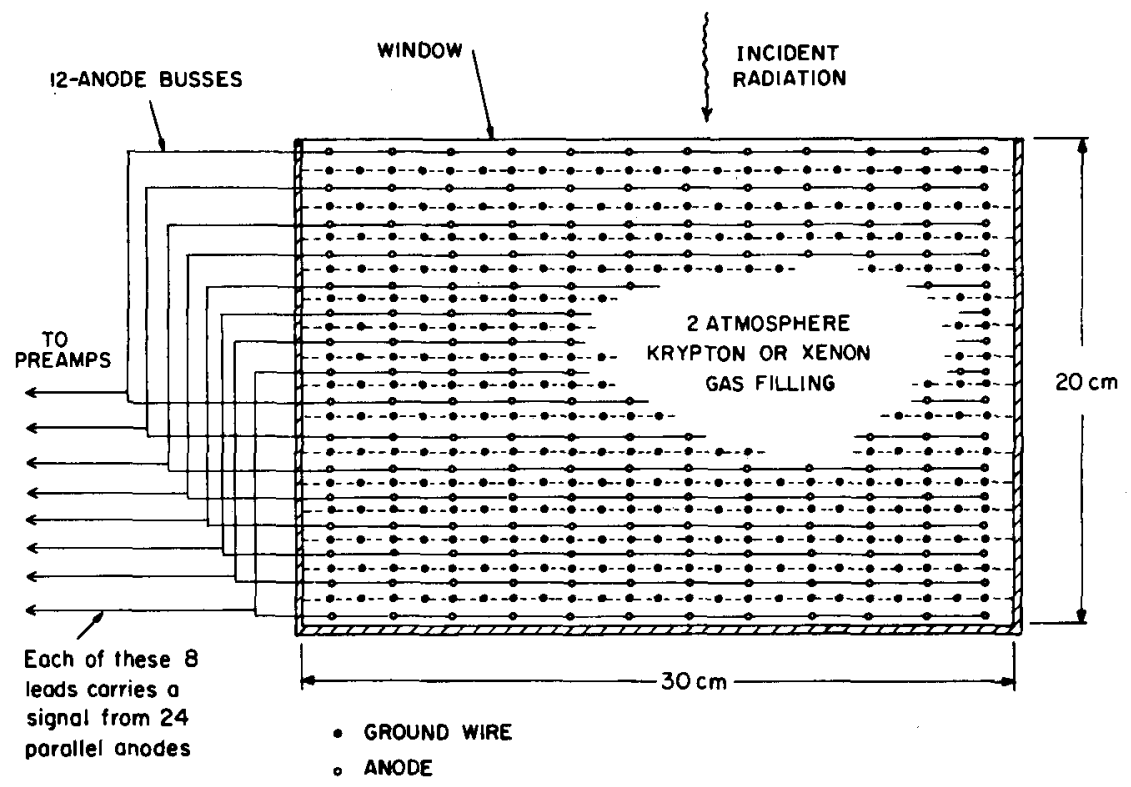

Fig. 2. Cross-section of the fluorescence-coincidence proportional counter developed by Stein and Lewin (1967).

At M.I.T. an interesting detector is in preparation by Stein and Lewin (1967). Their 'multi-electrode fluorescence-coincidence gas proportional counter' is a vessel, $20 \mathrm{~cm}$ deep with a surface of $30 \times 60 \mathrm{~cm}$. It will be filled with krypton or xenon at a pressure of 2 atmospheres (Figure 2). A characteristic signature for the detection of a photon with energy above or near to the K-edge of the detector gas (krypton: $14 \mathrm{keV}$; xenon: $35 \mathrm{keV}$ ) can be obtained in the form of the detection of the coincident occurrence of 2 events in 2 different cells. One of these events is the photoelectric ejection of a $\mathrm{K}$ electron by an incident X-ray, producing a pulse at one of the anodes. The other event is the capture of the fluorescent characteristic $\mathrm{K} \mathrm{X}$-ray photon of the detector gas itself, which may be emitted subsequent to the K photo process. Since the amplitude of the pulse resulting from this capture corresponds, within the limits of resolution of the counter, to the average K X-ray energy of the detector gas, the coincident detection of the two events constitutes a highly specific signature for a photon which has undergone a $\mathrm{K}$ photoelectric interaction. This signature is not easily forged by Compton electrons which result from interactions of high energy photons in the detector: these electrons are distributed nearly uniformly and isotropically in the detector gas, and produce pulses on many of the electrodes. In the energy range $15-80 \mathrm{keV}$ this kind of counter should detect about $25 \%$ of the incident flux from a celestial X-ray source, while rejecting over $96 \%$ of the background resulting from Compton interactions in the detector. 


\subsection{SCINTILLATION DETECTORS}

In the energy range above 20 to $50 \mathrm{keV}$, scintillation detectors are more efficient and are certainly a more reliable type of photon detector. Since photons of these energies penetrate rather deeply into the atmosphere, X-ray astronomy in this energy range may be performed by means of balloons. However, in that case sufficient precautions must be taken to eliminate the quite intense background of secondary X-rays resulting from cosmic ray debris in the atmosphere. With balloons, fairly long integration times are possible. An example of a simple but highly successful set-up is given in Figure 3, taken from Bleeker et al. (1967). The X-ray counter consists of a NaI (T1) crystal, $76 \mathrm{~mm}$ in diameter by $5 \mathrm{~mm}$ thick. The crystal photomultiplier assembly is mounted in a cylindrical tin cup ( $2 \mathrm{~mm}$ thickness) which provides an effective shielding against background $X$-rays in the energy range concerned. The collimator consists of two coaxial cones of tin ( $2 \mathrm{~mm}$ thick) with a half angle at the top of $18^{\circ}$, which corresponds to the zenith angle of Cyg XR. 1 at its meridian passage at the latitude of the observer $\left(52^{\circ} \mathrm{N}\right)$. The inner surface of the cup and the collimator is covered with copper foil $(0.3 \mathrm{~mm}$ thick) in order to absorb $\mathrm{K} \mathrm{X}$-rays which are possibly generated in the tin. The maximum effective area seen by the source is $26 \mathrm{~cm}^{2}$. A plastic scintillator guard counter surrounds the detector and the collimator for the rejection of background events associated with charged particles. A rotating tin disk ( $3 \mathrm{~mm}$ thick) in
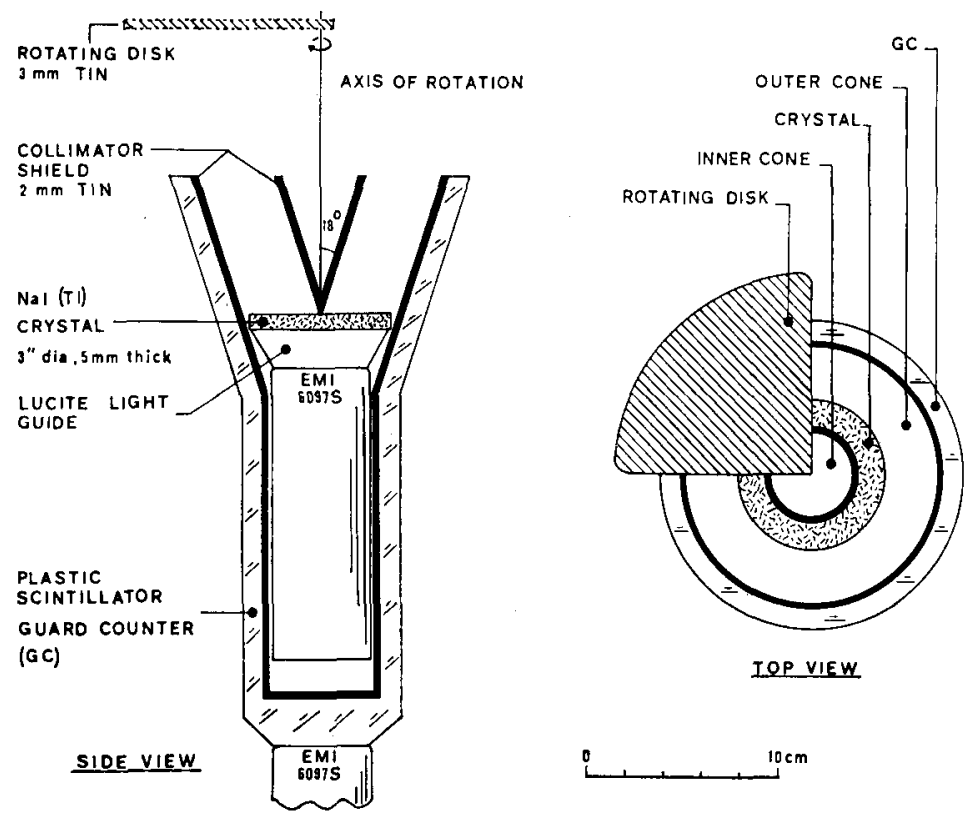

FIG. 3. Configuration of the X-ray telescope used by the Leiden Group for the measurements of the hard X-ray spectrum of Cygnus XR-1 (Bleeker et al., 1967). 
front of the detector covers a part of the opening angle and revolves around the axis of the collimator. The period of rotating is about $100 \mathrm{sec}$. Therefore, the disk periodically masks the X-ray source during the time it is in the field of view. Similar detectors with all kinds of minor variations have flown or are going to be flown in rockets and satellites.

\section{Image-Forming Techniques}

For wavelengths longer than a few Angströms imaging instruments can be based on glancing incidence reflecting or on diffraction. For shorter wavelengths this is impossible, as explained in Section 1, and mechanical collimators must be applied. In Section $3 \cdot 1$ we discuss the Fresnel zone plate technique; in Section 3.2 grazing incidence techniques, and in Section 3.3 mechanical collimators.

\subsection{THE FRESNEL-SORET ZONE PLATE}

In this device convergence of the incident rays is produced by diffraction at a series of concentric circular diaphragms. If $r_{1}$ is the diameter of the inner zone, the diameter of the $m$ th zone is:

$$
r_{m}=r_{1}^{2} m^{1 / 2}
$$

Furthermore, the focal distance of the first order image is:

$$
f=r_{1}^{1 / 2} / \lambda \text {. }
$$

With the Fresnel-Soret zone plate a theoretical resolution should be attainable, comparable to the diameter of the outermost transparent ring. High-quality zone plates are now produced at the Physics Laboratory at Tübingen University by Möllenstedt and at the Technical University of Delft, Holland, by Le Poole; see Figure 4.

However, there are drawbacks to the zone plate technique:

(1) The area of the zone plate is limited by the focal distance and the wavelength; for instance, for a focal distance of $1 \mathrm{~m}$ and a wavelength of $50 \AA$ and a zone plate consisting of 100 rings, $r_{1}$ is $0.07 \mathrm{~mm}$ and the largest diameter $r$ is $0.7 \mathrm{~mm}$. The distance between the two outermost rings is 3.5 micron. So, the light gathering power of this device is small, but the resolution would be 3.5 micron.

(2) The second drawback is the chromatic effect: light of different wavelengths converges at different distances; a careful predispersing or filtering of the incident light is necessary to produce chromatically purer images.

(3) Finally, a considerable part of the light, about $30 \%$, passes as zero-order light, giving an appreciable background haze. This difficulty could be overcome if means could be found to subtract the zero-order image from the first order one by using a suitable pinhole (Einighammer, 1966). 


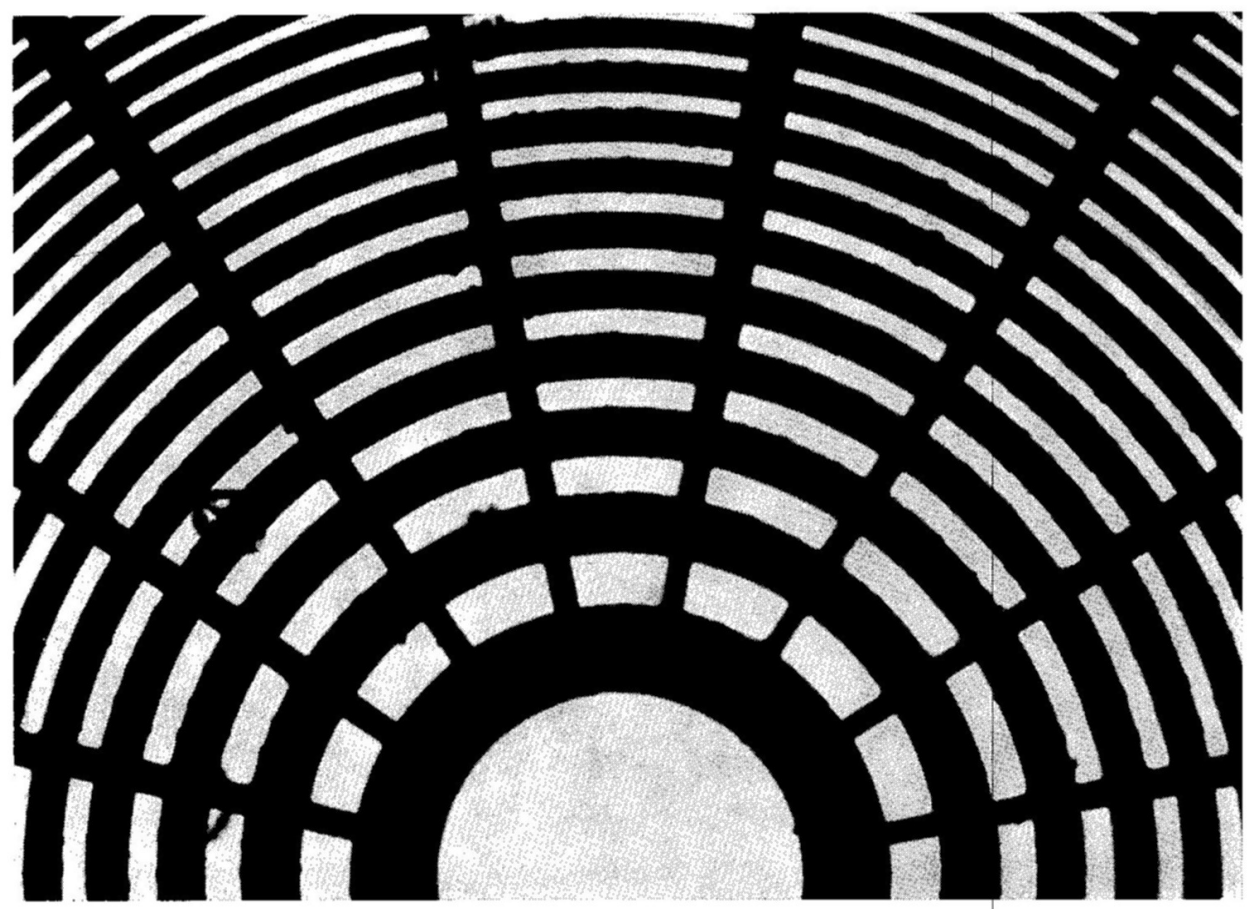

FIG. 4. Fresnel-Soret zone plate produced at the Technical University of Delft by Le Poole and co-workers; the diameter of the part of the zone plate reproduced here is about $0.3 \mathrm{~mm}$.

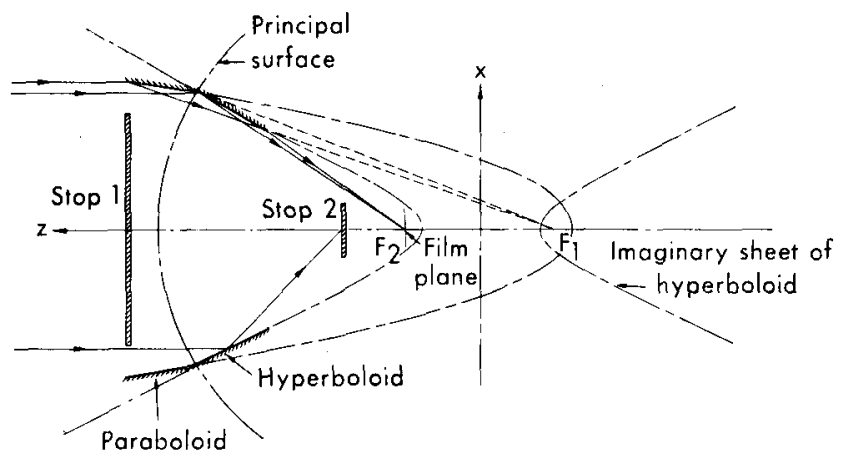

FIG. 5. Paraboloid-hyperboloid glancing-incidence solar X-ray telescope used by Underwood and Muney (1967).

\subsection{GLANCING INCIDENCE OPTICS}

Considerable advance in the development of glancing incidence optics has been made by the American Science and Engineering Group (Giacconi et al., 1965a, b) and later by the Goddard Space Flight Center Group with Underwood and Muney (1967). With a view to the smallness of the angles of incidence, extreme care must be taken to 
have a very good polish of the surface, a problem that now seems well solved by the GSFC-group. The instrument makes use of a combined paraboloid-hyperboloid surface while at relevant places stops are introduced to avoid the vignetting encountered in earlier models (see Figure 5). Reference is also made to the paper by Reidy et al. (1968), who showed that the paraboloid-hyperboloid surfaces can successfully be approximated by conical surfaces.

\subsection{MECHANICAL COLLIMATORS}

The simplest kind of mechanical collimator is the cellular or the honeycomb collimator, see e.g. Figure 6. The example given in Figure 7 collimates on a slit at the sky with a narrowest width of about 1.5 at half maximum. However, the width in the other direction is much larger, of the order of 10 to $15^{\circ}$, depending on the construction. This collimator, which has been used extensively by the ASE-group, has been successfully applied in the first X-ray scans of the sky.

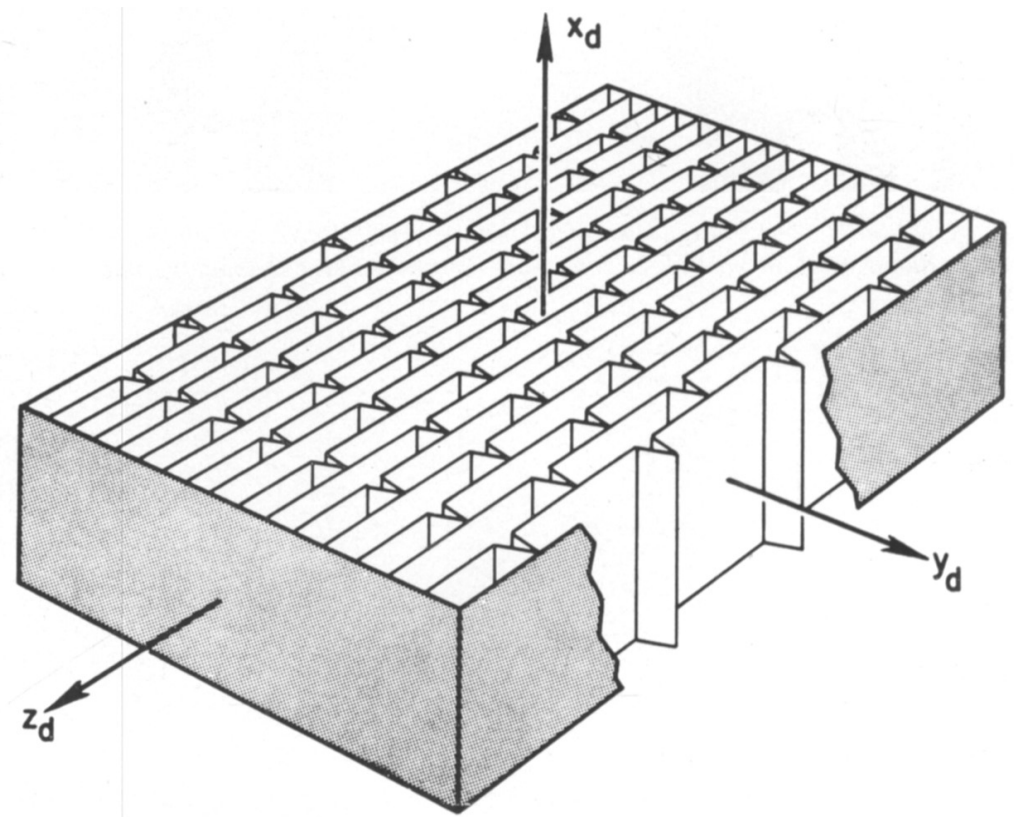

Fig. 6. Schematic drawing of a cellular collimator, after Rossi (1966).

A more sophisticated type of collimator that also scans the sky in a slit mode is the modulation collimator, also called the Oda collimator, after its designer. The principle is illustrated in Figure 7. It consists essentially of two plane grids placed one in front of the other at a suitable distance. The diameter of the wires is nearly equal to the spacing between them. In a parallel beam of rays (left-hand figure) the front grid casts a sharp 

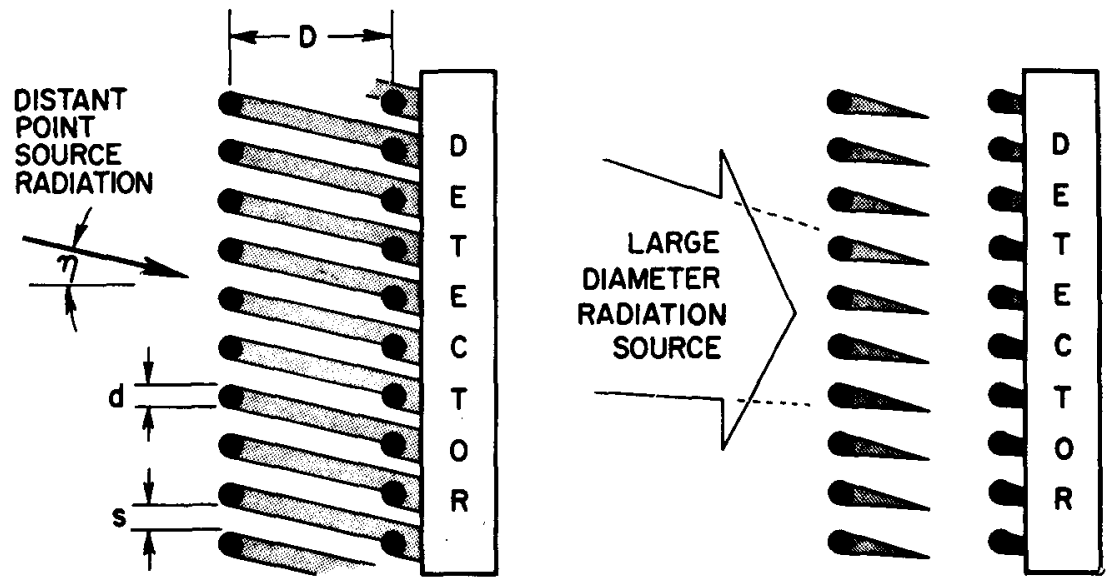

FIG. 7. Illustrating the principle of operation of the modulation collimator. The drawing on the left indicates the shadowing that obtains with parallel radiation; at the right-hand side the situation is illustrated in the case of a large diameter radiation source. After B. Rossi (1966).

shadow on the rear grid. The shadow shifts as the orientation of the collimator relative to that of the incident beam changes. The transmission of the collimator changes correspondingly, being a maximum when the shadow of the front wires falls exactly on the backwires and a minimum when it is centered half way between adjacent wires. For a source with a large diameter the illumination of the detector remains always the same (right-hand part of Figure 7). Hence, this collimator is very well suited for determining diameters of cosmic X-sources; collimators with a resolving power finer than $1^{\prime}$ are feasible (see e.g. Rossi, 1966).

Of course the system can be extended and brought to a greater degree of refinement: a number of the wire units of the Oda collimator can be placed behind each other in order to have a better resolution in determining the angular size and the location of celestial X-ray sources; this technique has been used successfully by the ASE-MITgroup, who could reduce the angular resolution in one direction to some $10^{\prime \prime}$. Further experiments of this kind using stellar pointing rockets have been flown by the ASEgroup and are planned for further flights (Gursky et al., 1967).

A further step forward may be introduced by the technique of the sieve-plates in development at the Utrecht Space Research Laboratory, after a suggestion of Le Poole from Delft. The sieve-plates are identical plates containing holes with diameters to be chosen at will from about 20 micron to about 200 micron. The holes are placed in a rectangular pattern at mutual distances depending on their sizes but ranging between 30 and 300 micron. By placing a series of these sieve plates behind each other at well chosen distances a very high collimating accuracy may be attained. The Utrecht Space Research Laboratory is planning to apply this technique in a solar X-ray limbscanning spectrograph to be flown in the ESRO-TD2 satellite (Heynekamp), in a 
rocket solar X-ray high-dispersion spectrophotometer (Dijkstra) and in a rocket celestial X-ray telescope (Heynekamp). Laboratory studies indicate that a pointing accuracy and an angular resolution of the order of $20^{\prime \prime}$ or perhaps somewhat better may be attainable. Technically difficult is the problem of the alignment of the plates.

\section{X-ray Spectrographs}

A variety of X-ray spectrographs have been flown in the last few years; all of these were applied to solar spectrography. Reference is made to published or forthcoming papers by various groups, mostly in the Astrophysical Journal, such as those by Hinteregger, Manson, the NLR-group, Pounds et al., Neupert. In principle, two techniques have been applied. Diffraction gratings used in glancing incidence mode now allow photographing the solar spectrum down to a wavelength of a few tens of Angströms. At shorter wavelengths spectral resolution may be obtained by using the well-known property of crystals that the angles of incidence and of emergence are equal for a beam

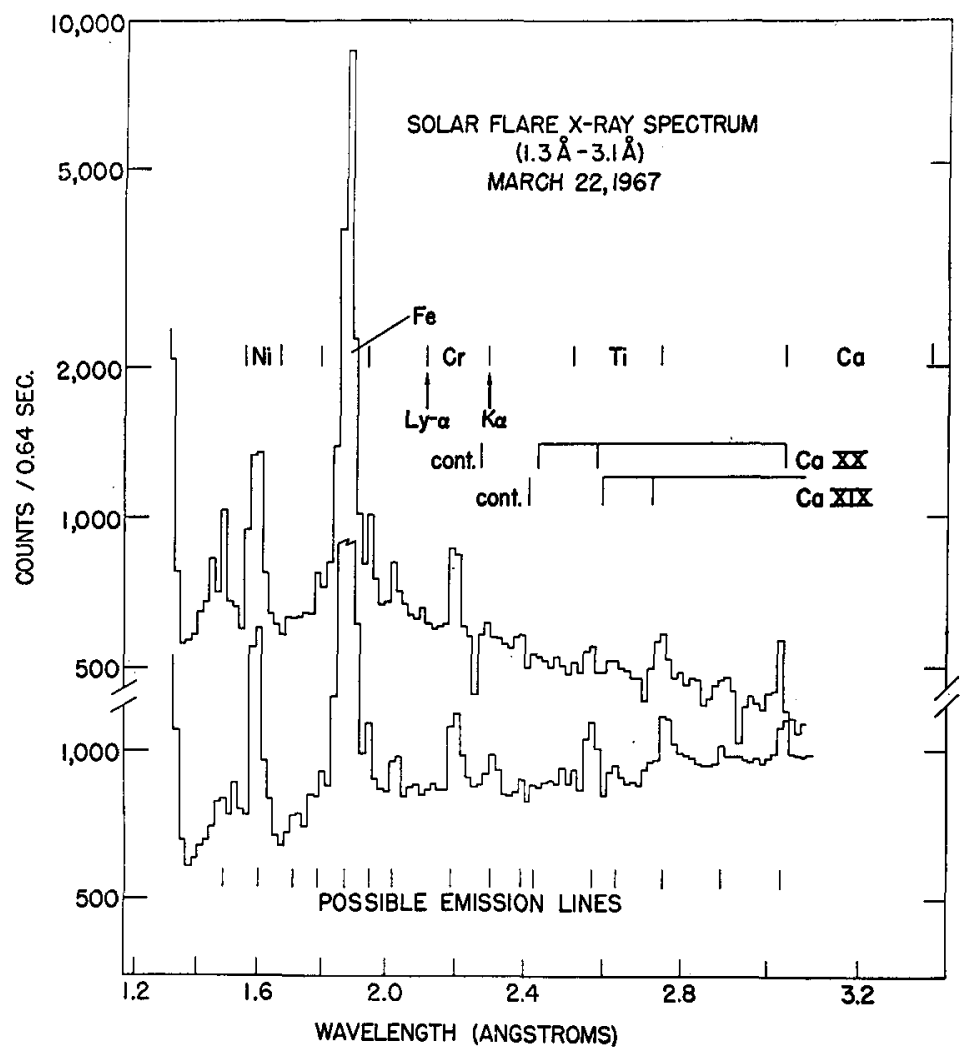

FIG. 8. The solar spectrum between 6.3 and 20 A observed by the OSO-III instrument during a flare on March 22, 1967, and on previous days when no flare was in progress. After Neupert et al. (1967). 


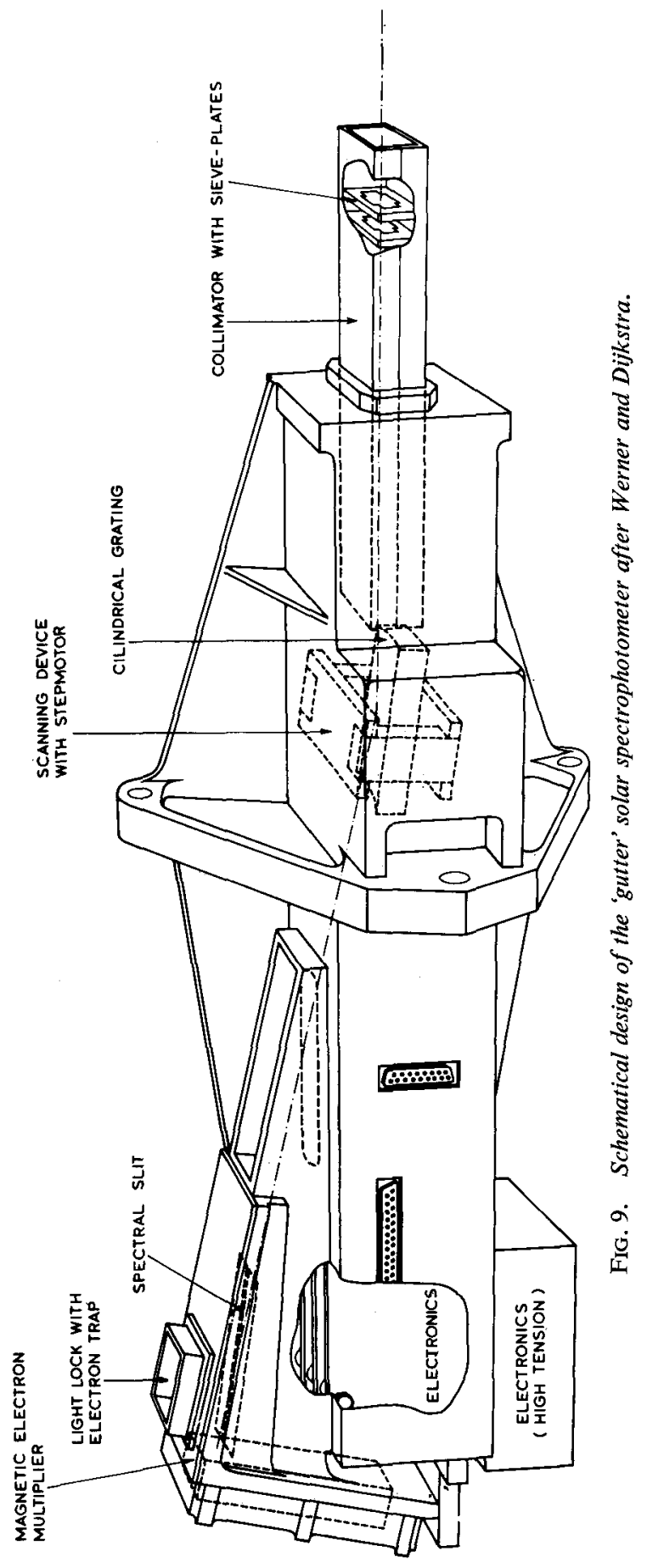


of parallel light and that a simple relation exists between the wavelength and this angle. Hence, a crystal acts as a very sharp monochromator in the X-ray region. By turning the crystal with reference to the incident light beam a spectral scanning is obtained. This technique has been applied mostly for wavelengths between about 5 and $30 \AA$, but has recently been extended by Neupert et al. (1967) down to $1.3 \AA$ (Figure 8). In that region, and particularly at shorter wavelengths, spectral discrimination may be further obtained by pulse-height discrimination with proportional counters or scintillation counters.

A new type of solar X-ray spectrograph, under development, is the 'gutter' spectrograph, developed at the Technical University of Delft and the Utrecht Space Research Laboratory by Werner and Dijkstra. The 'gutter' spectrograph makes use of a cylindrical concave grating with the grooves parallel to the cylinder axis. The grating is used in glancing incidence, but in a quasi-normal mode. As shown in Figure 9, solar radiation is collimated by a sieve-plate collimator in such a way that the plane through

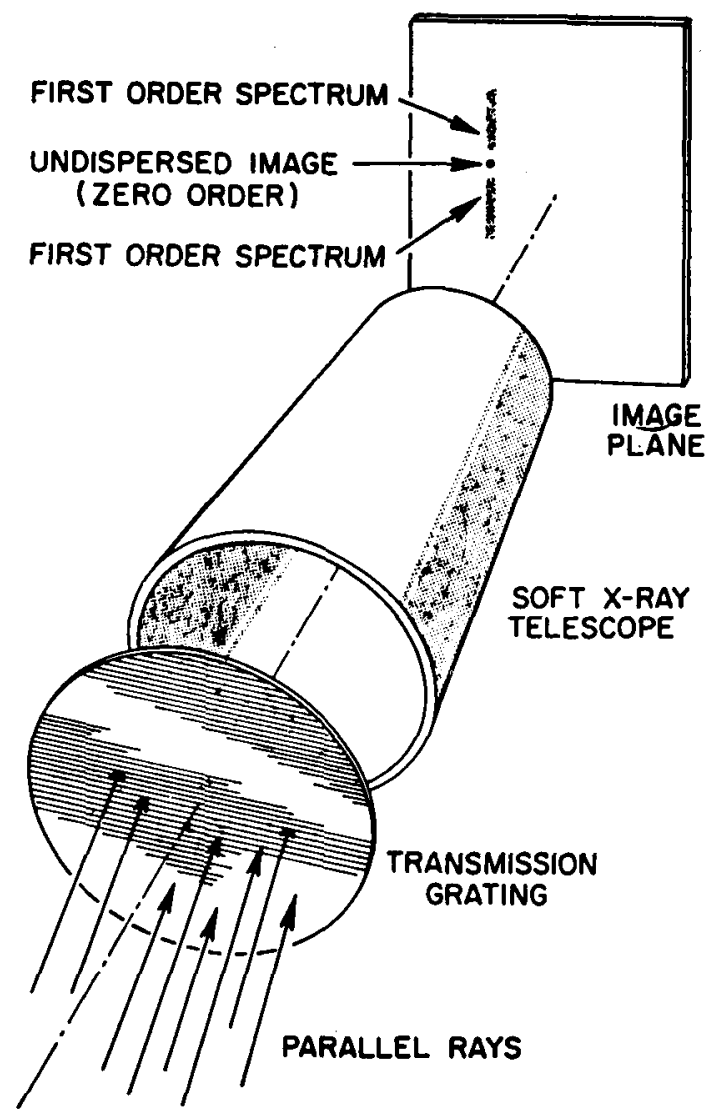

FIG. 10. Principle of the soft X-ray slitless spectrometer of Gursky and Zehnpfennig (1966). 
the axis of the grating and the vector representing the direction of the incident radiation are normal to the grating. The spectrum then is formed at some distance from the grating above it where the relevant part of the spectrum is selected by the exit slit. Spectral scanning is obtained by slowly turning the grating around an axis coincident wiht the central groove.

A proposal for an image-forming slitless spectrometer for soft X-ray astronomy has been published by Gursky and Zehnpfennig (1966) and worked out by Zehnpfennig (1966). The set-up is very much like the objective grating as used in classical astrophysics, and consists of the combination of a soft X-ray transmission grating and an image-forming soft X-ray telescope, the grating being positioned in front of the entrance aperture of the telescope in order to disperse a portion of the incident radiation before it is collected by the telescope. See Figure 10.

An essential difficulty was the production of the transmission grating. The required structure was a picket-fence type of grating consisting of parallel strips of material of low transmission to the wavelength range of interest, separated by strips of relatively high transmission material. A grating constant of $10^{-4} \mathrm{~cm}$ or smaller was required to achieve reasonable dispersion in the first-order spectrum. The applied process (Zehnpfennig, 1966) consists of evaporating a metal such as gold on a thin plastic replica grating at near grazing incidence such that the metal is deposited preferentially in certain regions of each groove. See Figure 11.

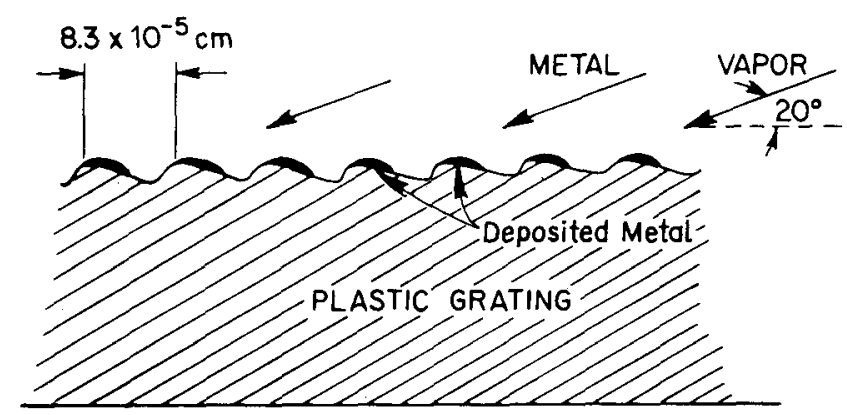

FIG. 11. Fabrication of a soft X-ray transmission grating after Zehnpfennig (1966); metal is vapor deposited on a thin replica grating at near grazing incidence.

The X-ray telescope used in this spectrometer has a focal length of $84 \mathrm{~cm}$, a diameter of $7.6 \mathrm{~cm}$, a collecting area of $2 \mathrm{~cm}^{2}$ and a nominal angular resolution of $1 \mathrm{~min}$ of arc. Laboratory spectra obtained with this instrument show the zero-order central image of the source and spectral lines with a width at half height of the order of about $0.5 \AA$. The instrument should be applicable to point sources such as the cosmic X-ray sources. However, a very long integration time and the photographic technique are needed. 
Perhaps a large proportional counter aboard a stellar pointing rocket would yield the same result in a shorter interval of time.

\section{X-ray Spectroheliograph}

We briefly mention the ASE X-ray spectroheliograph, in preparation for the Apollo Telescope Mount (Giacconi, 1967). The instrument should be capable of the following achievements during a 56-day mission:

(a) To obtain images of X-ray flare events with a spatial resolution of the order of a few seconds of arc.

(b) To simultaneously record flare spectra over the range of 2 to $10 \AA$ with a spectral resolution of a fraction of an $\AA$. These observations should be continued during the development of the event and correlated with ground observations. The instrument should be able to obtain solar images with fine spatial resolution during non-flare conditions at selected wavelength intervals. In the Apollo Telescope Mount the instrument is man-operated for coarse pointing while fine pointing is done automatically. The instrument has a length of $280 \mathrm{~cm}$ and consists of two concentric, con-focal optical elements. The larger of the two elements has an approximate inside diameter of $31 \mathrm{~cm}$; the smaller element has an inside diameter of about $23 \mathrm{~cm}$. The axial length of these elementsi s $35 \mathrm{~cm}$. There is another, smaller mirror with a focal length of $93 \mathrm{~cm}$ and an inside diameter of $8 \mathrm{~cm}$. It is $15 \mathrm{~cm}$ long. The images formed by the two large mirrors are recorded by the camera; in addition there is an objective for visible light which also produces an image for the exact location of the features. Also this image is recorded by the camera. The image formed by the small mirror is displayed electronically on a cathode ray tube. The camera will contain $300 \mathrm{~m}$ of film of $70 \mathrm{~mm}$ format. The camera will operate in either automatic or manual mode; the film magazine should be replaced every 14 days. One of the two shutters will be used for the X-ray image and the second for the visible light image. The camera will record on film the undispersed image and the X-ray spectrum focussed on the film plane by the large $X$-ray mirror. When filters are substituted for the diffraction grating by controlled action of the astronaut during non-flare conditions, the camera will record broad-band images of the Sun in selected regions of the X-ray spectrum. Simultaneously, on the same film visible light images from the achromatic lens will also be recorded. These images will be used to determine the orientation on the Sun of the X-ray images. In addition, during active periods, there will be visible features such as sunspots which will determine roll orientation.

\section{Acknowledgement}

Gratefully I acknowledge the help of many friends and colleagues at the Utrecht Space Research Laboratory and abroad, for commenting on a first draft of the manuscript and for sending me their preprints in advance of publication. 


\section{References}

Austin, G., Giacconi, R., Gursky, H., Kellogg, E., Kutz, M., Payne, P., Sinnamon, G., Tietsch, R., Waldron, J., Waters, J. (1967) Amer. Sci. Engineering no. 1567.

Bleeker, J.A. M., Burger, J. J., Deerenberg, A. J. M., Scheepmaker, A., Swanenburg, B. N., Tanaka, Y. (1967) Astrophys. J., 147, 391.

Boyd, R.L.F. (1965) Space Sci. Rev., 4, 35.

Culhane, J.L., Herring, J., Sanford, P. W., O'Shea, G., Phillips, R.D. (1966) J. Sci. Instrum., 43, 908.

Einighammer, H.J. (1966) Naturwiss., 53, 272.

Giacconi, R., Harmon, N. F., Lacey, R.F., Szilagnyi, Z. (1965a) J. Opt. Soc. Amer., 55, 345.

Giacconi, R., Reidy, W.P., Zehnpfennig, T., Lindsay, J.C., Muney, W.S. (1965b) Astrophys. J., $142,1274$.

Giacconi, R. (1967) Experimental Interface Requirements Document for the Apollo Telescope Mount, Experiment S-054 for X-ray Spectrographic Telescope, ASE 1607.

Gursky, H., Zehnpfennig, T. (1966) Appl. Opt., 5, 875.

Gursky, H., Giacconi, R., Gorenstein, R., Manko, H., Waters, J.R. (1967) A Program of High Angular Resolution Studies of Celestial X-Ray Sources, NASA C-R 752.

Mayer, U. (1964) Space Sci. Rev., 3, 781.

Neupert, W.M., Gates, W., Swartz, M., Young, R. (1967) Astrophys. J., 149, L 79.

Reidy, W.P., Vaiana, G.S., Zehnpfennig, T., Giacconi, R. (1968) Astrophys. J. (in press).

Rossi, B. (1966) in Perspectives in Modern Physics. Ed. by R.E. Mashale, Interscience Publ., New York, p. 383.

Stein, J.A., Lewin, W.H.G. (1967) Rev. of Sc. Instrum. (in press).

Underwood, J.H., Muney, W.S. (1967) Solar Phys., 1, 129.

Zehnpfennig, T. (1966) Appl. Opt., 5, 1855.

\section{DISCUSSION}

R. Wilson: I want to inquire about the 'gutter' grating spectrograph. What is the spectral range that can be covered, and what is the spectral resolution?

C. de Jager: In our first flight we would like to cover the spectral range between 100 and $200 \AA$, with a spectral resolution which we hope to be approximately $0.3 \AA$ or perhaps $0.5 \AA$. This grating will have 1200 lines per millimeter. In a next flight we want to cover the range between 40 and $65 \AA$. 